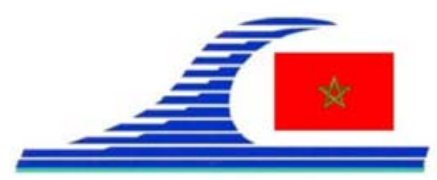

Conférence Méditerranéenne Côtière et Maritime EDITION 2, TANGER, MAROC (2011)

Coastal and Maritime Mediterranean Conference

Disponible en ligne - http://www.paralia.fr - Available online

\title{
Cycle de reproduction de la moule Mytilus galloprovincialis Lamarck 1819 au Maroc
}

\author{
Sanaa BHABY ${ }^{1}$, Oum Kelthoum BELHSEN ${ }^{2}$, Ahmed ERRHIF ${ }^{1}$
}

1. Université Hassan II, Casablanca, Laboratoire d'Ecologie Aquatique et Environnement, Maroc.

s.bhaby@yahoo.fr

2. Institut National de Recherche Halieutique, Laboratoire de pathologie des coquillages, Casablanca, Maroc.

\section{Résumé :}

L'étude du cycle de reproduction de la moule Mytilus galloprovincialis a été réalisée au niveau de deux sites situés sur le littoral atlantique marocain: Cap Beddouza et Imessouane. Pour cela un échantillonnage mensuel a été réalisé sur une période de deux ans depuis mars 2009 jusqu'au mars 2011. L'étude histologique et pondérale (indice de condition) des cellules folliculaires a montré que l'activité de la gamétogénèse est bien développée tout au long de l'année chez la population soumise à un upwelling permanent, alors qu'elle est annuelle chez la population marquée par un upwelling temporaire. Cependant les deux populations présentent une rareté de stade de repos sexuel (en été) et sont caractérisées par un synchronisme pour le stade d'émission principale des gamètes.

Mots-clés :

Mytilus galloprovincialis - Reproduction - Imessouane - Indice de condition upwelling - Cap Beddouza

\section{Introduction}

Mytilus galloprovincialis est une espèce des zones à distribution géographique lusitanienne. Elle représente la seule espèce de Mytilus localisée au Maroc, et ce depuis le quaternaire ; et comme la moule africaine Perna perna, elle se répartit le long des côtes marocaines méditerranéenne et atlantique.

Sa période de reproduction n'est pas synchronique et varie de la fin du printemps jusqu'à la fin d'automne. Elle est liée à plusieurs facteurs dont la température et l'abondance de la nutrition. Malgré son caractère saisonnier son cycle de reproduction peut avoir des variations spatio-temporelles considérables. La présence de zones de remontée d'eau froide (upwelling) le long des côtes marocaines modifie le caractère tempéré des eaux du littoral et perturbe ainsi le déroulement normal du cycle saisonnier caractéristique des zones tempérées. Cette étude a pour objectif la détermination de la variation des phases du cycle de reproduction et sa rythmicité chez deux populations de Mytilus galloprovincialis. 
La connaissance de la Mer :

un vecteur du développement durable en Méditerranée

\section{Matériels et méthodes}

Le premier site d'étude se situe au niveau du Cap Beddouza (zone atlantique centrale). Le deuxième site est situé au niveau d'Imessouane (zone atlantique sud). Il est influencé par un upwelling variable au cours de l'année. Un échantillonnage mensuel a été réalisé sur deux ans (mars 2009 - mars 2011). Chaque échantillon est composé de 30 animaux. Ils sont collectés à marée basse. Les températures ont été enregistrées à $30 \mathrm{~cm}$ de profondeur. Différentes mesures ont été effectuées avant la dissection des animaux (poids total, poids de la coquille, le poids de la chair). Le sexe a été déterminé à partir de l'examen des coupes histologiques du manteau. L'évolution de l'activité gonadique au cours du cycle sexuel a été déterminée par la méthode de LUBET (1959). L’indice gonadique a été déterminé à partir des coupes histologiques (SEED, 1975).

\section{Résultats}

A Cap Beddouza la durée des phases de la gamétogénèse ne montre pas de différence entre les deux années étudiées contrairement aux phases d'arrêt de cette gamétogénèse (figure 1). Les gonades présentant les stades de dégénérescence des cellules sexuelles et d'arrêt du redémarrage de la gamétogénèse sont observées entre mai et juillet. La phase de repos sexuel n’a été observée qu'en 2010. Ces phases finales du cycle sexuel coïncident avec la période de la régénération de tissu conjonctif du manteau. La gamétogénèse s'initie surtout entre juillet et octobre lorsque le tissu conjonctif est plein de réserves. Puis elle continue à se manifester à faible pourcentage jusqu'à l'hiver. Durant cette saison la prévitéllogénèse et la spermiation sont maximales. La gamétogénèse présente un ralentissement important entre août et septembre 2009, et entre septembre et octobre 2010. A Imessouane l'émission des gamètes se fait durant toute l'année et les phases du repos sexuel sont rares. L'examen de la gonade a montré que le développement est continu dans le temps, ce qui peut être expliqué par l'asynchronisme des stades de reproduction. Au sein de la même coupe histologique, il est souvent observé deux stades successifs. Pour les deux populations, les émissions principales des gamètes se situent en hiver et se poursuit an printemps.
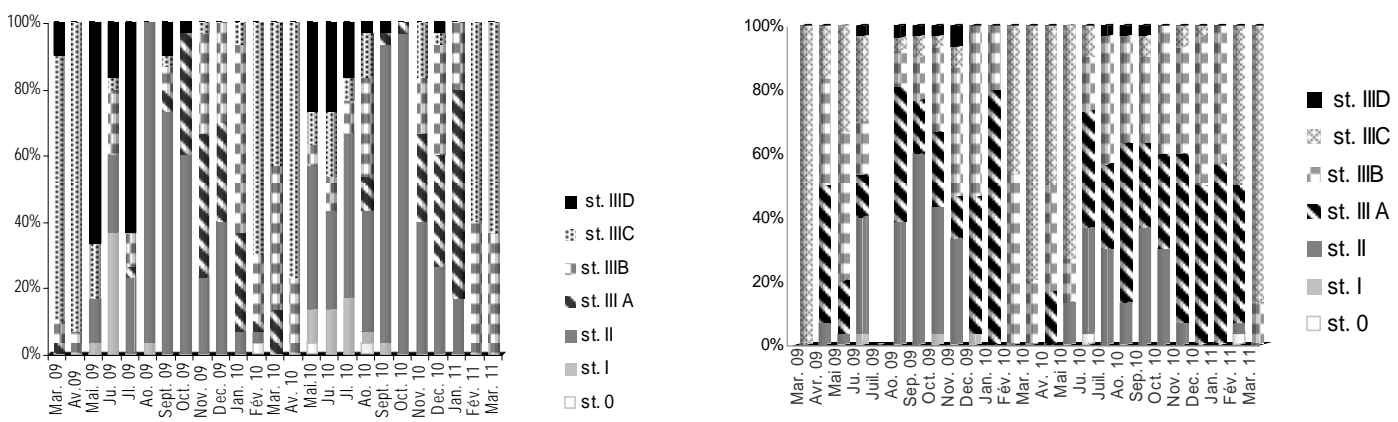

Figure 1. Evolution temporelle des stades de reproduction au niveau de Cap Beddouza et à Imessouane. 
L'indice gonadique montre que la gamétogénèse est active tout au long de l'année chez la population d'Imessouane, alors qu'il montre une diminution à partir du mois de mai jusqu'en juillet chez celle de Cap Beddouza (figure 2). A la fin de l'été et au cours de l'automne, cet indice a des valeurs assez stables autour de 2 reflétant une phase de développement de l'activité gamétogénétique. Les deux populations deviennent plus matures à partir de la fin de l'automne et au début de l'hiver.

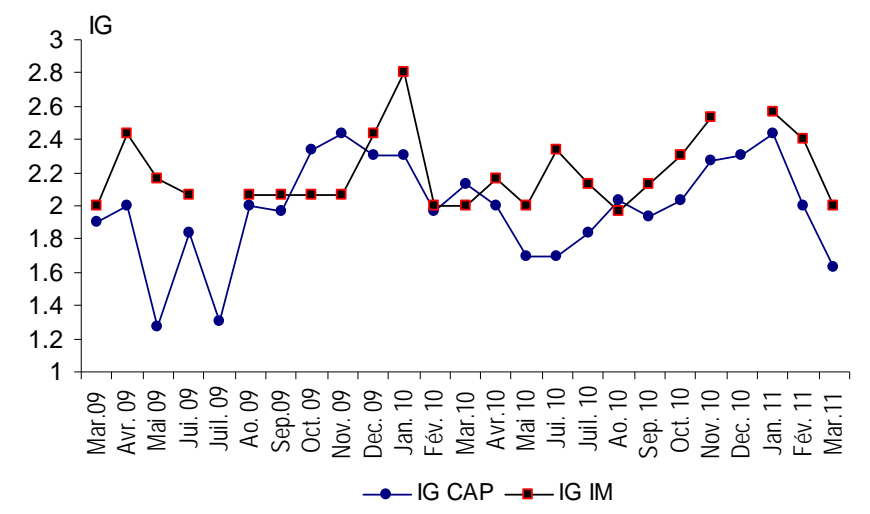

Figure 2. Variations de l'indice gonadique (CAP : Cap Beddouza ; IM : Imessouane).

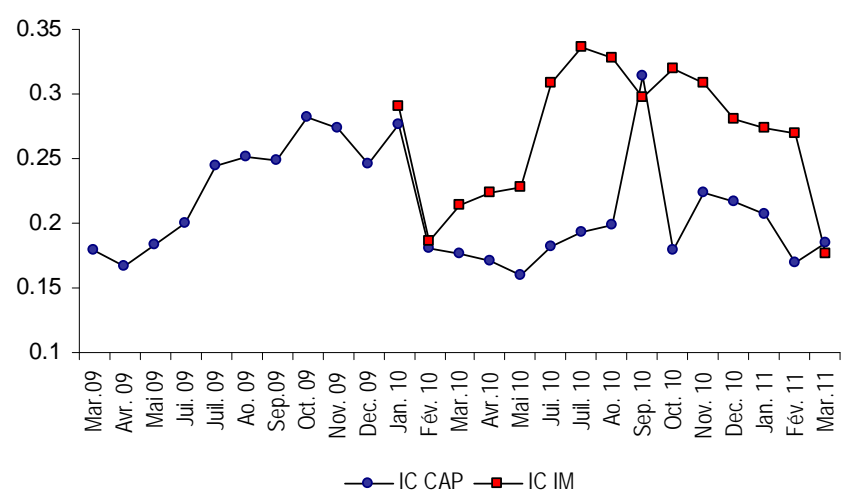

Figure 3. Evolution de l'indice de condition (CAP : Cap Beddouza ; IM : Imessouane).

L'évolution de l'indice de condition montre que la plage de la variation annuelle est plus forte à Cap Beddouza par rapport à Imessouane (figure 3). Les deux populations perdent beaucoup de leur poids entre février et mai. A partir du mois de juin l'indice de condition commence à augmenter. L'augmentation maximale du poids chez la population d'Imessouane coïncide avec la chute de la température provoquée par la remontée des eaux profondes. 
La connaissance de la Mer :

un vecteur du développement durable en Méditerranée

\section{Discussion}

L'étude du cycle de reproduction de Mytilus galloprovincialis à Cap Beddouza et à Imessouane montre une activité asynchrone de la gamétogénèse. Ce cycle est annuel au niveau de Cap Beddouza alors qu’il est continu à Imessouane. Les températures enregistrées dans les deux sites restent dans la plage thermique supérieure, déclenchant l'arrêt du cycle de reproduction. L'état du repos sexuel, n'est observé que rarement, en été ; alors que la moitié de sa prévalence est provoquée par une forte infestation par le trématode Proctoeces. Ces résultats corroborent ceux obtenus par IDHALLA (1997). La rythmicité et la durée des différentes phases varient de façon significative entre les deux populations. L'émission des gamètes est continue toute l'année à Imessouane mais avec un taux plus élevé au printemps, période de ponte à Cap Beddouza. La population de ce site montre une deuxième période d'émission moins importante à la fin de l'automne. Ceci montre qu'en dehors des périodes de pontes principales on a des pontes partielles. Ces résultats concordent avec ceux de VILLALBA (1995) et IDHALLA (1997). L'indice de condition n'est pas toujours un paramètre fiable pour estimer les variations du poids en relation avec le cycle reproducteur. Les variations du poids observées sont liées à l'accumulation des composés biochimiques qui sont sous l'influence directe de la richesse trophique du milieu. L'utilisation de l'indice de condition reste toutefois intéressante. Ces variations confirment la période de ponte maximale, où le manteau devient très mince sans cellule sexuelle et sans réserve. Il a ainsi indiqué que la période d'accumulation des réserves se produit après la période de remontée d'eau froide. Il a aussi signalé la période principale de la maturité des gamètes.

\section{Références bibliographiques}

IDHALLA M. (1997). Etude de la biologie des moules Perna perna Linné (1758) et Mytilus galloprovincialis Lamark (1819) dans la baie d'Agadir. Thèse Univ. Agadir, $158 \mathrm{p}$.

LUBET P. (1959). Recherches sur le cycle sexuel et l'émission des gamètes chez les mytilidés et les Pectinidés. Thèse Univ. Paris. 159 p.

SEED (1975). Reproduction in Mytilus edulis L. (Moullusca: Bivalvia) in European waters. Pubbl. Stn. Zool. Napoli, 39, pp 317-334.

VILLALBA A. (1995). Gametogenic cycle of cultured mussel, Mytilus galloprovincialis, in bays of Galicia (N.W. Spain). Aquaculture, 130, pp 269-277. doi:10.1016/0044-8486(94)00213-8 